\title{
Concentrações de reguladores vegetais no estiolamento in vitro de ananás do campo
}

\section{Concentrations of plant regulators in the blanching in vitro of pineapple of the field}

\author{
Márcia Maria Dias ${ }^{1 *}$; Moacir Pasqual2; Aparecida Gomes Araújo ${ }^{3}$; Verônica \\ Andrade dos Santos ${ }^{4}$; Antônio Clarete de Oliveira ${ }^{5}$; Vantuil Antônio Rodrigues ${ }^{5}$
}

\section{Resumo}

O abacaxizeiro ornamental Ananas comosus (L.) Merr. var. ananassoides (Baker) Coppens \& F. Leal, também denominado de ananás do campo ou coroa verde, é uma planta ainda pouco explorada economicamente, apesar do potencial ornamental como "flor de corte". Estudos sobre a propagação do ananás do campo são escassos, neste sentido, a micropropagação desta, pode representar um avanço para a produção comercial da espécie. O objetivo do trabalho foi avaliar as diferentes combinações de 6-BAP e $\mathrm{GA}_{3}$ na multiplicação e desenvolvimento dos explantes de Ananas comosus (L.) Merr. var. ananassoides (Baker) Coppens \& F. Leal. Para a condução do experimento foram utilizados brotos de abacaxizeiro ornamental pré-estabelecidos in vitro. $\mathrm{O}$ delineamento utilizado foi inteiramente casualizado, em esquema fatorial $4 \times 4$, constituído de quatro concentrações de 6-BAP $(0 ; 0,25 ; 0,5$ e $\left.1,0 \mathrm{mg} . \mathrm{L}^{-1}\right)$ e quatro concentrações $\mathrm{GA}_{3}(0 ; 0,5 ; 1,0$ e 2,0 mg.L-1 $)$ combinadas. Os resultados obtidos indicaram que a interação $1,0 \mathrm{mg} . \mathrm{L}^{-1}$ de 6 -BAP e $0,5 \mathrm{mg} \cdot \mathrm{L}^{-1}$ de $\mathrm{GA}_{3}$ proporcionou maior número de brotações. A adição de 6-BAP propiciou uma redução do comprimento da parte aérea e do número de raízes. A adição de $\mathrm{GA}_{3}$ ocasionou um maior comprimento da parte aérea e não influenciou no enraizamento dos explantes.

Palavras-chave: Micropropagação. Abacaxizeiro ornamental. Bromeliaceae. Citocinina. Giberelina.

\begin{abstract}
The ornamental pineaple Ananas comosus (L.) Merr. var. ananassoides (Baker) Coppens \& F. Leal, also denominated of pineapple of the field or it crowns green it is a plant still little explored economically in spite of the ornamental potential as " cut flower ". Studies about the propagation of the pineapple of the field are scarce, in this sense, the micropropagation of this can represent a progress for the commercial production of the species. The objective of the work was to evaluate the different combinations of 6-BAP and $\mathrm{GA}_{3}$ in the multiplication and development of the explants of Ananas comosus (L.) Merr. var. ananassoides (Baker) Coppens \& F. Leal. The randomized experimental desing was used, with a factorial scheme $4 \times 4$ consisting of four concentrations of 6-BAP $\left(0 ; 0,25 ; 0,5\right.$ and $\left.1,0 \mathrm{mg} . \mathrm{L}^{-1}\right)$ and four concentrations of $\mathrm{GA}_{3}\left(0 ; 0,5 ; 1,0\right.$ and $\left.2,0 \mathrm{mg} \cdot \mathrm{L}^{-1}\right)$ combined. According to the results, the interaction
\end{abstract}

\footnotetext{
${ }^{1}$ Eng $^{\circ}$ Agr $^{\circ}$, Msc. Bolsista de Apoio Técnico. Universidade Estadual de Montes Claros, Unimontes, Rua Reinaldo Viana, 2630, Bico da Pedra, 39440-000, Janaúba, MG. E-mail: marciamaridias@yahoo.com.br

${ }^{2}$ Prof. Dr. Departamento de Agricultura, DAG, Universidade Federal de Lavras, UFLA, Caixa Postal 3037, 37200-000, Lavras, MG. E-mail: mpasqual@ufla.br

${ }^{3}$ Pesquisadora EMDAGRO, Laboratório Sarah Brandão-Sergipe Tec, Av. Dr. Carlos Rodrigues da Cruz, s/n, Capucho, 49.081-190, Aracaju, SE.E-mail: agaraujo2003@hotmail.com

${ }^{4}$ Eng $^{\circ}$ Agr $^{\circ}$ Pós-doutoranda Agronomia/Fitotecnia. Universidade Federal de Lavras, UFLA. E-mail: veronicaandrad@yahoo.

${ }^{5}$ Laboratorista do Laboratório de Cultura de Tecidos Vegetais DAG, UFLA. E-mail: mpasqual@ufla.br

* Autor para correspondência
} 
1,0 mg. $\mathrm{L}^{-1}$ of 6 -BAP and $0,5 \mathrm{mg} . \mathrm{L}^{-1}$ of $\mathrm{GA}_{3}$ provided larger sprouts number. The addition of 6-BAP propitiated a reduction of the bud length and of the roots number of explants. The addition of $\mathrm{GA}_{3}$ caused a larger length of the aerial part and it didn't influence in the rooting of the explants.

Key words: Micropropagation. Ornamental pineapple. Bromeliaceae. Cytokinin. Gibberellin

\section{Introdução}

Além das diversas espécies de abacaxizeiro utilizadas na alimentação, com grande valor econômico e nutricional, destacam-se os abacaxizeiros ornamentais pela relevante importância na ornamentação, em programas de melhoramento genético, ou ainda na produção de fibras (SILVA, 2006).

Dentre as espécies de abacaxizeiro com finalidade ornamental foram exportadas no Ceará aproximadamente US\$ 412,9 mil em 2004, para Holanda (70\%), EUA (12\%), Portugal (8\%) e Alemanha (5\%). As principais espécies de abacaxi ornamental comercializadas são Ananas comosus var. erectifolius, A. comosus var. bracteatus e $A$. comosus var. ananassoides (CARVALHO et al., 2009).

Esta diversidade genética do abacaxizeiro é importante no que se refere a novidades e busca por novas variedades para sustentar o mercado do segmento de flores (SOUZA et al., 2007a).

O Ananas comosus var. ananassoides, também denominado de ananás do campo ou coroa verde, apesar de serainda pouco explorado economicamente destaca-se entre as demais variedades, dadas as características para "flor de corte", ou melhor dizendo fruto, por apresentar genótipos de frutos pequenos com coloração amarelo creme a rosa e hastes ou pedúnculos longos (SOUZA et al., 2007b).

A obtenção das mudas por propagação assexuada pode ser realizada pelo método convencional, o qual permite a disseminação de patógenos, ou ainda, por meio da cultura de tecidos (ALBERT, 2004).

A cultura de tecidos, apesar do alto custo, proporciona a obtenção de milhares de mudas a partir de uma gema em pequeno intervalo de tempo e espaço. E o mais importante disto, é o fato desta técnica permitir a obtenção de plantas totalmente livres de pragas e doenças (ALBERT, 2004).

As citocininas na cultura de tecidos promovem a divisão celular (PASQUAL, 2004) e são indispensáveis para a quebra da dominância apical e na indução da proliferação de gemas axilares (ALBERT, 2004). Uma das principais citocininas utilizadas na micropropagação de plantas é a 6-benzilaminopurina (BAP) (PASQUAL, 2004), obtendo-se efeitos significativamente superiores no número de brotações (DINIZ et al., 2003). O excesso desta, no entanto, é tóxico e caracteriza-se pela redução no tamanho das folhas, encurtamento de entrenós, excessiva brotação (ALBERT, 2004) e até mesmo, promove inibição da brotação das gemas conforme observado por Qi-Guang et al. (1986) em Castanea mollissima.

As giberelinas constituem outro grupo de substâncias reguladoras vegetais conhecidas principalmente pelo seu efeito no alongamento de caules (ASSIS; TEIXEIRA, 1998). No entanto, poucas culturas in vitro mostram respostas às giberelinas (CALDAS; HARIDASAN; FERREIRA, 1998).

A principal giberelina utilizada na propagação é o $\mathrm{GA}_{3}$ (ácido giberélico) (PASQUAL, 2004) e o uso desta é uma alternativa para alongar partes aéreas quando estas ainda não estão em condições de serem individualizadas para o enraizamento, devido ao reduzido tamanho, conforme ob servado na cultura da Macela (Egletes viscosa (L.) Less.) por Diniz et al. (2003).

Diante disto, este trabalho objetivou avaliar as diferentes combinações de 6-benzilaminopurina (BAP) e ácido giberélico $\left(\mathrm{GA}_{3}\right)$ na multiplicação e desenvolvimento dos explantes de Ananas comosus var. ananassoides. 


\section{Material e Métodos}

Para a condução do experimento, brotos de abacaxizeiro ornamental pré-estabelecidos in vitro foram transferidos, sob condições assépticas em câmara de fluxo laminar, para placas de Petri contendo papel filtro. Sobre o papel, os brotos foram submetidos à retirada das folhas e raízes com o auxílio de pinça e bisturi para obtenção de explantes com aproximadamente $1,0 \mathrm{~cm}$ de comprimento.

Os explantes foram inoculados em meio de cultivo MS (MURASHIGE; SKOOG, 1962) suplementado com 30 g.L $\mathrm{L}^{-1}$ de sacarose, 5 g.L${ }^{1}$ de ágar, 0,1mg. $\mathrm{L}^{-1}$ de ácido naftaleno acético e conforme os tratamentos adicionados de diferentes combinações de 6-benzilaminopurina (BAP) e ácido giberélico $\left(\mathrm{GA}_{3}\right)$ com pH ajustado para 5,7. Antes da inoculação, os meios de cultura foram distribuídos em frascos contendo $50 \mathrm{~mL}$ de meio de cultivo, os quais foram identificados, vedados com tampas translúcidas de polipropileno e autoclavados durante 20 minutos à $121^{\circ} \mathrm{C}$ e $1,5 \mathrm{~atm}$.

O delineamento estatístico utilizado foi inteiramente casualizado (DIC) com 16 tratamentos constituídos de quatro concentrações de BAP (0; 0,$25 ; 0,5$ e 1,0 mg. $\left.\mathrm{L}^{-1}\right)$ combinadas com quatro concentrações de ácido giberélico $(0 ; 0,5 ; 1,0$ e 2,0 mg. $\mathrm{L}^{-1}$ ), com 4 repetições e cada parcela experimental representada por um frasco contendo 3 brotos (explantes).

Após a inoculação, os frascos foram mantidos por 45 dias no escuro para indução do estiolamento. Os brotos obtidos foram avaliados quanto ao número de brotações, comprimento da parte aérea e número de raízes.

Os dados foram submetidos à análise estatística pelo software SISVAR - Sistema de Análise de Variância de Dados Balanceados (FERREIRA, 2000). Inicialmente procedeu-se ao Teste $\mathrm{F}$ e posteriormente as médias quantitativas dos tratamentos em que houveram diferenças significativas foram comparadas entre si através da Regressão. Para efetuar a comparação entre as médias dos reguladores vegetais isoladamente utilizou-se o Teste de Tukey a 5\% de probabilidade.

\section{Resultados e Discussão}

Para a variável número de brotações, a interação dos fatores concentrações de BAP e $\mathrm{GA}_{3}$ foi significativa pelo testeF.Aorealizaro desdobramento do fator concentrações de $\mathrm{GA}_{3}$ obteve-se diferença significativa para as concentrações 0,$25 ; 0,5$ e 1,0 mg.L - $^{-1}$ de BAP (Figura 1). 


$$
\begin{aligned}
& Y_{\text {GA } 3+0,25 m g . L^{-1} \text { BAP }}=4,184716-3,701466 x+1,136432 x^{2} \quad R^{2}=0,8836 \\
& Y_{\text {Ga3 }+0,5 m g . L^{-1} \text { BAP }}=5,291750-12,061542+13,446875 x^{2}-4,093583 x^{3} R^{2}=1 \\
& Y_{\text {Ga3 }+1,0 m g . L^{-1} \text { BAP }}=1,87500+22,544500 x-3,757500 x^{2}+11,227000 x^{3} R^{2}=1
\end{aligned}
$$

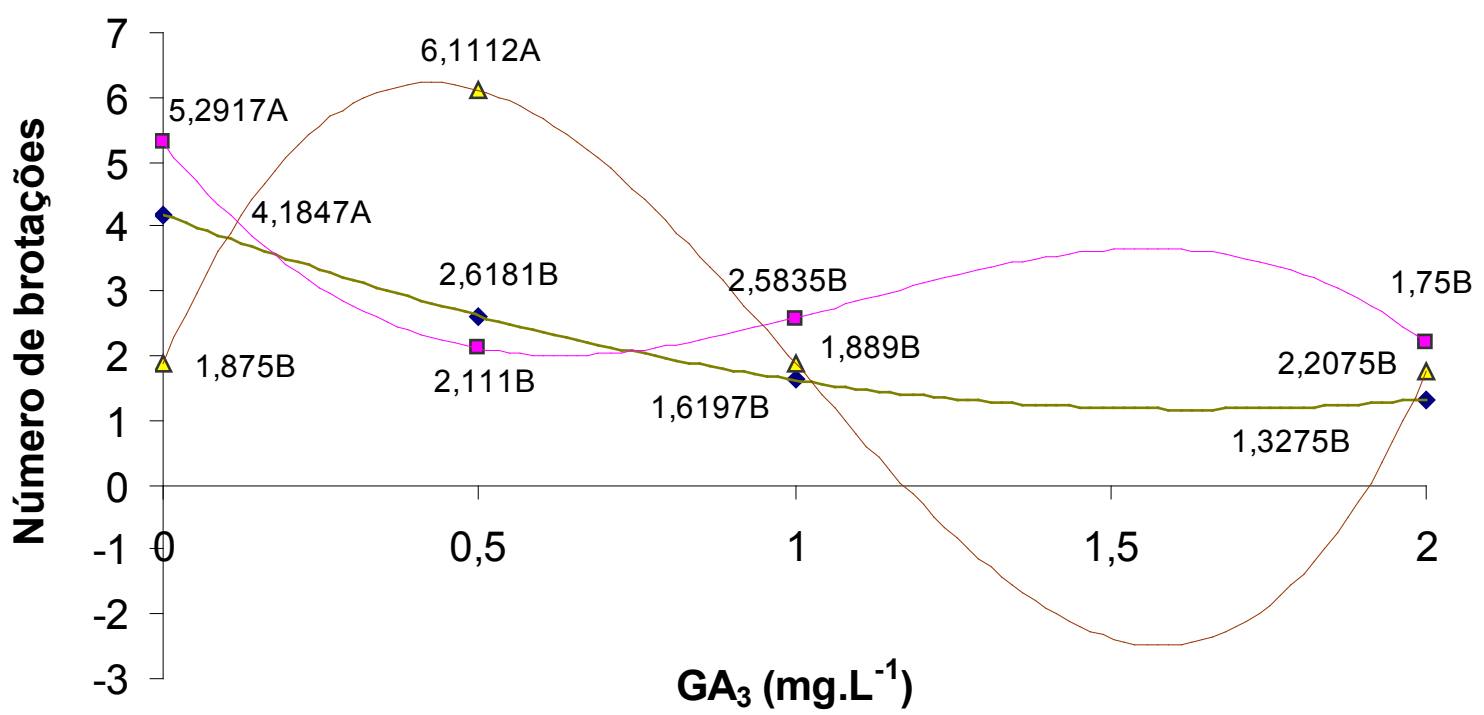

$0,25 \mathrm{mg} / \mathrm{L}$ BAP

$\square 0,5 \mathrm{mg} / \mathrm{L}$ BAP

$\triangle 1,0 \mathrm{mg} / \mathrm{L} \mathrm{BAP}$

Figura 1. Número de brotações por explantes de Ananas comosus var. ananassoides em função de diferentes concentrações de BAP e GA 3 em meio MS aos 45 dias de estiolamento. Lavras-MG, 2007.

O maior número de brotações para as concentrações de 0,25 e $0,5 \mathrm{mg} . \mathrm{L}^{-1}$ de BAP foi obtido na ausência do ácido giberélico que corresponderam a 4,42 e 5,29, respectivamente. Nestas concentrações de BAP, a concentração $0 \mathrm{mg}$. $\mathrm{L}^{-1}$ de ácido giberélico foi significativamente superior em relação às demais concentrações que não diferiram entre si pelo teste de Tukey ao nível de 5\% de probabilidade. No entanto, quando utilizou-se a concentração de 1,0 mg. $L^{-1}$ de 6-benzilaminopurina, o maior número de brotos foi obtido em associação com $0,5 \mathrm{mg}$. $\mathrm{L}^{-1}$ de ácido giberélico, com média de 6,11 brotos por explante, diferindo significativamente das demais concentrações. Em relação às concentrações de 0; 1 e 2 mg. $\mathrm{L}^{-1}$ de ácido giberélico combinado a concentração de 1,0 mg. $\mathrm{L}^{-1}$ de 6-benzilaminopurina não foram obtidas diferenças significativas entre estas no que diz respeito ao número de brotações.
Respostas semelhantes foram obtidas por Souza et al. (2005), na cultura in vitro de mandioquinhasalsa (Arracacia xanthorrhiza) sob intensidade luminosa de $110 \mu \mathrm{mol} . \mathrm{m}^{-2} \cdot \mathrm{s}^{-1}$, em que um maior número de brotações de 4,1 brotos foi observado com o aumento das concentrações de BAP para 0,4 mg. $L^{-1}$ na cultivar amarelo comum.

Carvalho et al. (1999) também observaram um acréscimo do número de brotos de Coffea arabica L. cv. catuaí, com o aumento das concentrações de BAP ao testar dosagens de até 12 mg.L - $^{-1}$ de BAP que correspondeu a aproximadamente 6 brotações aos 120 dias de cultivo in vitro sob intensidade luminosa de $16 \mu \mathrm{M} \cdot \mathrm{m}^{-2} \cdot \mathrm{s}^{-1}$.

Em ausência de luz, Moreira (2001) aos 40 dias de cultivo in vitro do abacaxizeiro cv. Pérola obteve 10,26 brotações em meio MS acrescido de 1,8 mg. $L^{-1}$ de ANA e 2,0 mg. $L^{-1}$ de BAP. Estes 
resultados foram superiores ao observado para a cultura do abacaxi-ornamental Ananas comosus (L.) Merr. var. ananassoides, possivelmente por se tratar de uma maior concentração, devendo-se levar em consideração também o fato de serem variedades diferentes.

Ao utilizar as concentrações 0,$0 ; 2,0 ; 4,0 ; 8,0$ e 16,0 mg. $\mathrm{L}^{-1}$ de $\mathrm{GA}_{3}$, Carvalho et al. (1999), obtiveram uma redução do número de brotações de Coffea arabica L. cv. catuaí com o acréscimo das dosagens em meio de cultivo MS. De acordo com o mesmo autor, a interação entre citocinina e ácido giberélico mostrou-se desfavorável, reduzindo consideravelmente o número de brotações por explante.

Quanto ao comprimento da parte aérea foi constatada diferença significativa para os fatores concentrações de BAP e $\mathrm{GA}_{3}$ isoladamente. Analisando o efeito das concentrações de BAP, a ausência de 6-benzilaminopurina promoveu maior comprimento $(8,45 \mathrm{~cm})$ em comparação às demais concentrações deste regulador vegetal (Figura 2).

$$
\begin{gathered}
Y=8,455313-40,548708 x+80,123750 x^{2}- \\
45,347667 x^{3} R^{2}=1
\end{gathered}
$$

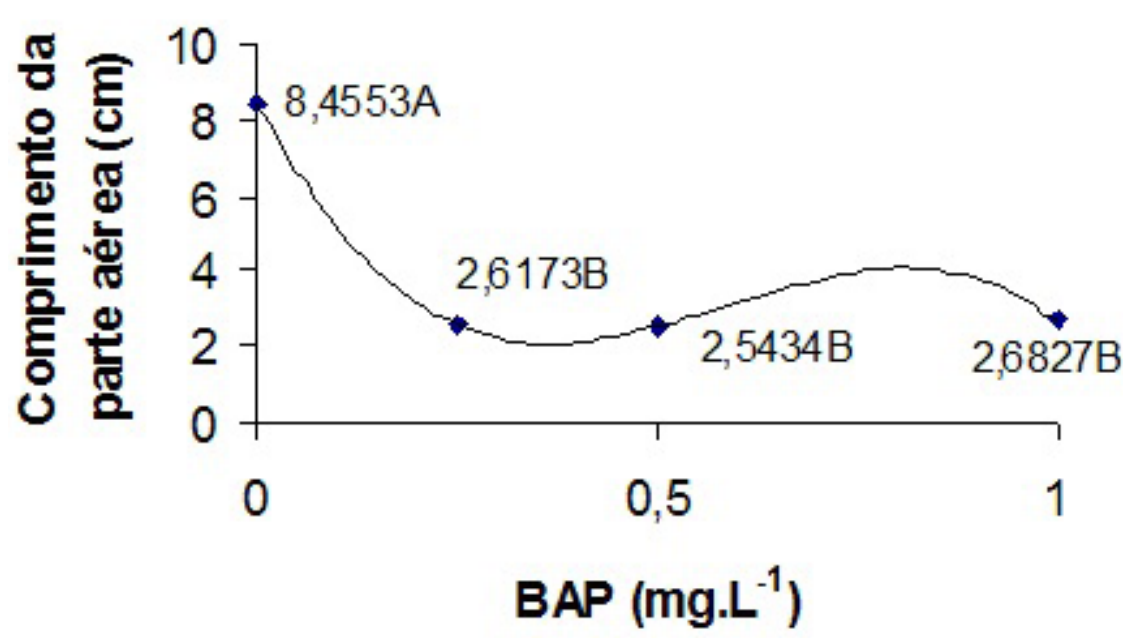

Figura 2. Comprimento da parte aérea de explantes de Ananas comosus var. ananassoides em função de diferentes concentrações de BAP em meio MS aos 45 dias de estiolamento. Lavras-MG, 2007.

O maior comprimento médio da parte aérea do explante em ausência de BAP foi significativamente superior ao obtido nas concentrações de 0,$25 ; 0,5$ e 1,0 mg.L $\mathrm{L}^{-1}$ de BAP, as quais não diferiram entre si ao nível de $5 \%$ de probabilidade pelo teste de Tukey .

Moreira (2001) também obteve maior comprimento médio dos brotos estiolados de abacaxizeiro cv. Pérola em meio MS desprovido de reguladores vegetais, os quais corresponderam a 1,$01 ; 2,6$ e 10,86 cm de comprimento aos 20, 40 e 80 dias de cultivo no escuro.
A ausência de BAP proporcionou a formação de plantas estioladas, com reduzido número de brotações e a medida em que aumentou-se a concentração deste regulador vegetal ocasionou-se uma redução da altura das plantas de mandioquinhasalsa (SOUZA et al., 2005).

Considerando-se a influência do ácido giberélico isoladamente sobre o comprimento da parte aérea do explante, melhores resultados foram obtidos com o acréscimo das doses até concentração de 2,0 mg...-1 de $\mathrm{GA}_{3}$ (Figura 3), que não diferiu significativamente das concentrações de 0,5 e 1,0 mg.L. $\mathrm{L}^{-1}$ de $\mathrm{GA}_{3}$ pelo teste de Tukey ao nível de $5 \%$ de probabilidade. 
Portanto recomenda-se a menor concentração de custo de produção de mudas. $0,5 \mathrm{mg} . \mathrm{L}^{-1}$ em função da possibilidade de reduzir o

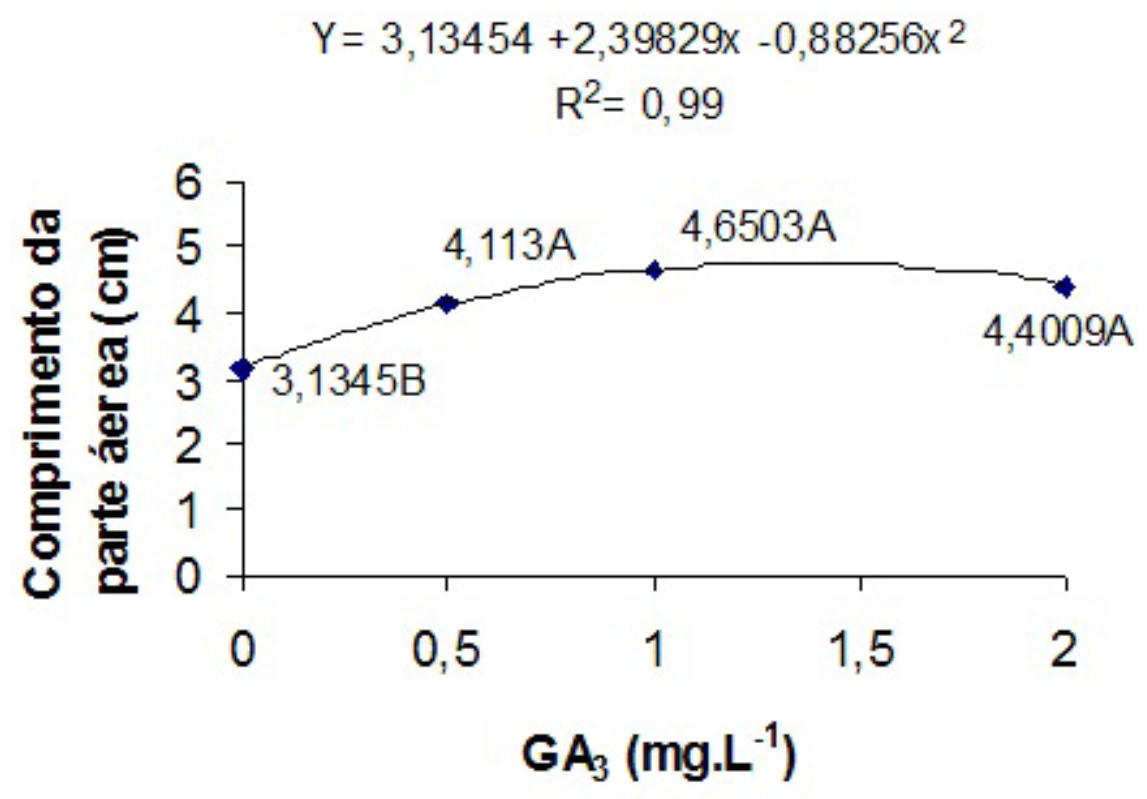

Figura 3. Comprimento da parte aérea de explantes de Ananas comosus var. ananassoides em função de diferentes concentrações de Ácido giberélico em meio MS aos 45 dias de estiolamento. Lavras-MG, 2007.

Souza et al. (2005), ao utilizar as concentrações de 0 a $0,25 \mathrm{mg} . \mathrm{L}^{-1}$ de $\mathrm{GA}_{3}$ no cultivo in vitro de mandioquinha-salsa, também observaram um maior comprimento médio das brotações com o acréscimo das concentrações deste regulador vegetal.

Um maior comprimento das plantas de Egletes viscosa foi verificado no tratamento com $0,5 \mathrm{mg} . \mathrm{L}^{-1}$ de $\mathrm{GA}_{3}$ na ausência de BAP com aproximadamente $4 \mathrm{~cm}$ de comprimento, aos 30 dias de cultivo in vitro, sob intensidade luminosa em torno de 2000 lux (DINIZ et al., 2003).

Quanto ao número de raízes apenas foi obtida diferença significativa para o fator concentrações de BAP. Os melhores resultados para esta característica foram obtidos na ausência de BAP conforme a (Figura 4), demonstrando uma redução deste número com a utilização deste regulador vegetal. 


$$
\begin{gathered}
Y=3,071938-21,434125 x+43,965500 x^{2}- \\
25,520000 x^{3} R^{2}=1
\end{gathered}
$$

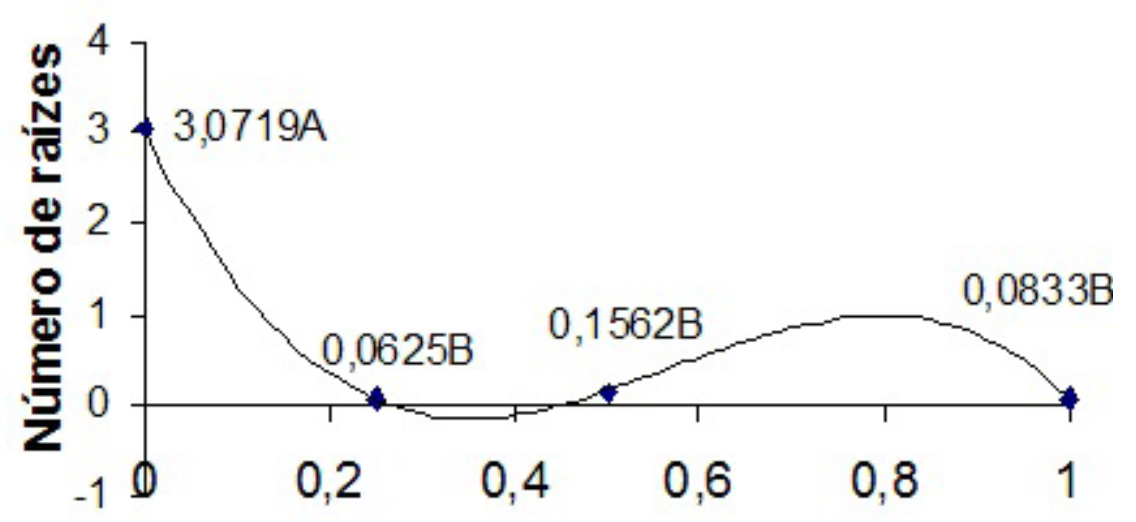

\section{$\operatorname{BAP}\left(\mathrm{mg} \cdot \mathrm{L}^{-1}\right)$}

Figura 4. Número de raízes de explantes de Ananas comosus var. ananassoides em função de diferentes concentrações de BAP cultivados em meio MS aos 45 dias de estiolamento. UFLA, Lavras-MG, 2007.

O maior número de raízes obtido correspondeu a uma média de 3,07 raízes por explante na ausência de BAP, o qual diferiu significativamente das médias observadas nas demais concentrações. Entre as médias resultantes dos tratamentos com acréscimo de 0,$25 ; 0,5$ e $1,0 \mathrm{mg} . \mathrm{L}^{-1}$ de BAP não houveram diferenças significativas quando comparadas entre si pelo teste de Tukey ao nível de 5\% de probabilidade.

De acordo com Diniz et al. (2003), no cultivo in vitro de Egletes viscosa, a adição de BAP promoveu uma redução no número de explantes com raízes. Em compensação houve um maior número de plantas com raízes quando foram utilizadas concentrações crescentes de GA 3 (DINIZ et al., 2003). Diferentemente, em mandioquinha-salsa a utilização desta giberelina até uma concentração de $0,25 \mathrm{mg} . \mathrm{L}^{-1}$ não proporcionou a formação de raízes nas plantas (SOUZA et al., 2005).

Isto se deve ao fato de que as espécies respondem diferentemente a aplicação de reguladores vegetais em função do balanço endógeno.

Para obtenção de uma eficiente produção de mudas in vitro é imprescindível priorizar a aquisição de um maior número de brotações, uma vez que, o principal objetivo é produzir o maior número de plantas possível, no menor espaço de tempo (GRATTAPAGLIA; MACHADO, 1998).

\section{Conclusões}

1) Para o cultivo do abacaxi-ornamental Ananas comosus var. ananassoides a interação $1,0 \mathrm{mg} \cdot \mathrm{L}^{-1}$ de BAP e 0,5 mg. $\mathrm{L}^{-1}$ de $\mathrm{GA}_{3}$ proporcionou maior número de brotações.

2) A adição de BAP propiciou uma redução do comprimento da parte aérea e do número de raízes.

3) O meio MS suplementado de $\mathrm{GA}_{3}$ ocasionou um maior comprimento da parte aérea e não influenciou no enraizamento dos explantes.

\section{Agradecimentos}

Os autores agradecem ao CNPq e a Fapemig pelo apoio financeiro no desenvolvimento da pesquisa.

\section{Referências}

ALBERT, L. H. de B. Aspectos morfo-anatomicos 
de mudas de abacaxizeiro 'Smooth cayenne' micropropagadas. 2004. Tese (Doutorado em Agronomia) - Universidade Federal de Lavras, Lavras.

ASSIS, T. F.; TEIXEIRA, S. L. Enraizamento de plantas lenhosas. In: TORRES, A. C.; CALDAS, L. S.; BUSO, J. A. (Ed.). Cultura de tecidos e transformação genética de plantas. Brasília: Embrapa/CNPH/CBAB, 1998. v. 1, p. 261-296.

CALDAS, L. S.; HARIDASAN, P.; FERREIRA, M. E. Meios nutritivos. In: TORRES, A. C.; CALDAS, L. S.; BUSO, J. A. (Ed.). Cultura de tecidos e transformação genética de plantas. Brasília: Embrapa/CNPH/CBAB, 1998. v. 1, p. 87-132.

CARVALHO, A. C. P. P.; PINHEIRO, M. V. M.; DIAS, G. M. G.; MORAIS, J. P. S. In vitro multiplication of ornamental pineapple by shoot etiolation and regeneration. Horticultura Brasileira, Campinas, v. 27, n.1, p. 103-108, jan./mar. 2009.

CARVALHO, G. R.; PIO, R.; PASQUAL, M.; CARVALHO, G. R.; SCARANTE, M. J. Efeitos do ácido giberélico e benzilaminopurina no desenvolvimento de plântulas de cafeeiro in vitro. Revista da Universidade de Alfenas, Alfenas, v. 5, n. 2, p. 185-187, 1999.

DINIZ, J. D. N.; ALMEIDA, J. L.; TEIXEIRA, A. L. de A.; GOMES, E. S.; HERNANDEZ, F. F. F. Ácido giberélico (GA3) e 6-benzilaminopurina (BAP) no crescimento in vitro de macela [egletes viscosa (1.) less.]. Ciência e Agrotecnologia, Lavras, v. 27, n. 4, p. 934-938, jul./ago. 2003.

FERREIRA, D. F. Analises estatísticas por meio do Sisvar para windows versão 4.0. In: REUNIÃO ANUAL DA REGIÃO BRASILEIRA DA SOCIEDADE INTERNACIONAL DE BIOMETRIA, 45., 2000, São Carlos. Anais... São Carlos: UFS Car, 2000. p. 255-258.

GRATTAPAGLIA, D.; MACHADO, M. A. Micropropagação. In: TORRES, A. C.; CALDAS, L. S.; BUSO, J. A. (Ed.). Cultura de tecidos e transformação genética de plantas. Brasília: Embrapa/CNPH/CBAB, 1998. v. 1, p. 183-260.

MOREIRA, M. A. Produção e aclimatização de mudas micropropagadas de abacaxizeiro Ananas comosus (L) Merril cv. Pérola. 2001. Tese (Doutorado em Agronomia) - Universidade Federal de Lavras, Lavras.

MURASHIGE, T.; SKOOG, F. A revised medium for rapid growth and bioassays with tobacco tissue cultures. Physiologia Plantarum, Copenhagen, v. 15, n. 6, p. 473497, June 1962.

PASQUAL, M. Propagação de plantas ornamentais. Lavras: UFLA/FAEPE, 2004. 106 p.
QI-GUANG, Y; READ, P. E.; FELLMAN, C. D.; HOSIER, M. A. Efect of cytokinin, IBA and rooting regime on Chinese chestnut culture in vitro. Hort Science, Alexandria, v. 21, n. 1, p. 133-134, feb. 1986.

SILVA, A. B. Biorreator e luz natural na micropropagação do abacaxizeiro. 2006. Tese (Doutorado em Agronomia) - Universidade Federal de Lavras, Lavras, MG.

SOUZA, F. V. D.; CABRAL, J. R. S.; SOUZA, E. H. de; SANTOS, O. S. N.; SANTOS-SEREJO, J. A. dos; FERREIRA, F. R. Caracterização morfológica de abacaxizeiros ornamentais. Magistra, Cruz das Almas, v. 19, n. 4, p. 319-325, out./dez. 2007 a.

SOUZA, F. V. D.; CABRAL, J. R. S.; SOUZA, E. H. de; SANTOS, O. S. N.; SANTOS-SEREJO, J. A. dos; FERREIRA, F. R.; SILVA, M. de J. da. Abacaxi ornamental: uma riqueza a ser explorada. Cruz das Almas: Embrapa/Mandioca e Fruticultura Tropical, dez. 2007b. (Boletim Técnico, n. 37). Disponível em: $<$ http:// www.cnpmf.embrapa.br/publicacoes $>$. Acesso em: 12 mar. 2011.

SOUZA, F. V. D.; MADEIRA, N. R.; TEIXEIRA, J. B.; ARIMURA, C. T.; JUNQUEIRA, C. S. Influência da concentração de $\mathrm{BAP}$ e $\mathrm{AG}_{3}$ no desenvolvimento in vitro de mandioquinha-salsa. Horticultura Brasileira, Brasília, v. 23, n. 4, p. 982-985, out./dez. 2005. 